\title{
Timely diagnosis of congenital heart disease - did we improve?
}

\author{
Andrea Keuffer, Jean-Pierre Pfammatter \\ Center for Congenital Heart Disease, University Hospital Berne, Switzerland
}

\section{Summary}

Introduction: Delayed recognition of congenital heart disease (CHD) is not infrequent and may have a negative impact on the child's prognosis. A study from our institution published in 2001 showed a rate of late diagnosis as high as $10 \%$ of all relevant $\mathrm{CHD}$ requiring therapy, and this rate was equal in cyanotic and acyanotic CHD.

Methods: A study identical to the one 12 years ago was performed with prospective evaluation of the time of first diagnosis of CHD during a 3-year period ending in June 2011. Only CHD that required surgical or catheterbased treatment was included. Late diagnosis was defined as diagnosis of CHD after discharge from the birth hospital for cyanotic CHD; for acyanotic CHD it was defined as first diagnosis at a time when therapy should already have taken place in accordance with recommended standards or if immediate treatment was necessary at the time of CHD recognition. In between the two studies came the recommendation of a nationwide neonatal pulse oximetry screening, starting in 2006.

Results: A total of 209 patients were included, $41 \%$ of these had cyanotic and $59 \%$ had acyanotic CHD. According to the entry criteria, late diagnosis was observed in 21 patients ( $10 \%$ of total population); $6 \%$ of cyanotic $\mathrm{CHD}$ patients (5 of 85 ) and $13 \%$ of acyanotic CHD patients (16 of 124). The two most frequently missed CHDs were atrial septal defect and coarctation (seven and six patients, respectively). Delayed diagnosis resulted in one death (unrecognised interrupted aortic arch in one neonate). Compared with the historical study in our referral population the main finding was that the total number of late diagnoses remained stable at $10 \%$, with a slight decrease only in the rate of late diagnosed cyanotic CHD (from 10 to $6 \%$ ) but with a rise in late diagnosed acyanotic CHD from 10 to $13 \%$ of all patients observed.

Conclusions: After 12 years of referring physician education and the implementation of a nationwide neonatal pulse oximetry screening, the rate of late diagnosis of $\mathrm{CHD}$ remained unchanged at $10 \%$ of all patients with only the rate of cyanotic CHD showing a slight decline.

Key words: congenital heart disease; late diagnosis; prognosis

\section{Introduction}

For most congenital heart disease (CHD) there is a consensus as to what would be the optimal timing for corrective intervention, allowing for the best possible prognosis of the affected children. For a long time liter- ature has dealt with delayed diagnosis of even critical CHD [1, 2], and in recent years there was still substantial published evidence of the negative impact on outcome due to late diagnosis of relevant CHD [3-6].

In our referral population a study was conducted and published in 2001, showing the rate of late diagnosis of CHD to be $10 \%$ without any difference between cyanotic and acyanotic CHD in the rate of late detection [7]. These results led to intensified education of referring physicians (focused clinical workshops for the referring paediatricians every 2 years since publication of the first study), and in 2006 a nationwide recommendation for neonatal pulse oximetry was implemented in our country [8]. The current study evaluated whether these measures led to a decline in the rate of children with CHD being treated late because of missed diagnosis.

\section{Methods}

The study was identical to that done in our historical cohort [7], with a prospective evaluation covering a 3-year period ending in June 2011. The timing of first diagnosis of all CHD patients was registered. Only patients with haemodynamically relevant CHD, with necessity for either surgical or catheter-based therapy were included. Children with CHD but without an indication for therapy and solely on observation on an outpatient basis were excluded. Patients admitted for reoperation or reintervention were excluded, as were patients referred from other institutions for treatment.

Patients with late diagnosis in cyanotic CHD were defined as newborns discharged from their birth clinic without a diagnosis of heart disease. For acyanotic CHD, late diagnosis was defined as first diagnosis of the defect at a time when, according to recommended standards [9], treatment should already have taken place, or if at the time of diagnosis immediate therapeutic action was required.

Echocardiographic screening in foetal life in our country is done by the caring obstetrician twice in pregnancy. Pregnant women are referred to a tertiary 
centre for further evaluation by a specialised foetal cardiologist only if primary screening revealed suspect findings.

\section{Results}

During the timespan covered by the evaluation, a total of 209 patients with newly diagnosed relevant CHD were prospectively included in this study. Eighty-five of the patients had cyanotic CHD and 124 children acyanotic CHD. About half of the patients were diagnosed in the early neonatal period (57\%) while 51 of the patients (24\%) were diagnosed at an age later than the neonatal period extending up to the age of 15 years. Especially pathologies of the aorta and atrial septal defect had a trend towards higher age at diagnosis of CHD. For a contemporary study there was an astonishingly low rate of in-utero diagnosis of only 19\% (39 children).

Table 1 gives an overview of the individual diagnoses encountered and the respective proportion of late diagnosis for each entity.

Late diagnosis of CHD was observed for the group of cyanotic patients in 5 of 85 patients (5.9\%) and in 16 of 124 acyanotic patients (12.9\%, table 2). For the entire study population the rate of late diagnosis was $10 \%$, the same level as in our historical patient cohort, where it was $9.9 \%$. As shown in table 2, a mild decline in the rate of late diagnosis was seen for the cyanotic patients (without statistical significance owing to the low number of index patients with late diagnosis), as was to be expected because of the introduction of the neonatal pulse oxymetry screening in Switzerland in 2006. Out of the five infants with late diagnosis in cyanotic $\mathrm{CHD}$ two born in private clinics did not have neonatal pulse oxymetry screening, two had it correctly done and in the last patient it was incorrectly done on the right arm instead of the leg.

For the patients with acyanotic CHD there was even a mild rise in the rate of late diagnosis from $10 \%$ in the first study period to currently $12.9 \%$, and this was especially due to underdetection of atrial septal defects and coarctation.

A few typical clinical findings that were not detected adequately were seen in the patients who were referred late: fixed split heart sounds in atrial septal defect were clinically present in seven patients. Absent or weak

Table 2: Proportion of patients with late diagnosis.

\begin{tabular}{lcc}
\hline Late diagnosis & $\begin{array}{l}\text { Study period I } \\
1996-1999\end{array}$ & $\begin{array}{l}\text { Study period II } \\
2008-2011\end{array}$ \\
\hline All patients with CHD & $32 / 323(9.9 \%)$ & $21 / 209(10 \%)$ \\
\hline Cyanotic CHD & $7 / 72(9.7 \%)$ & $5 / 85(5.9 \%)$ \\
\hline Acyanotic CHD & $25 / 251(10 \%)$ & $16 / 124(12.9 \%)$ \\
\hline Comparison
\end{tabular}

Table 1: Spectrum of cardiac malformations and proportion of patients with late diagnosis.

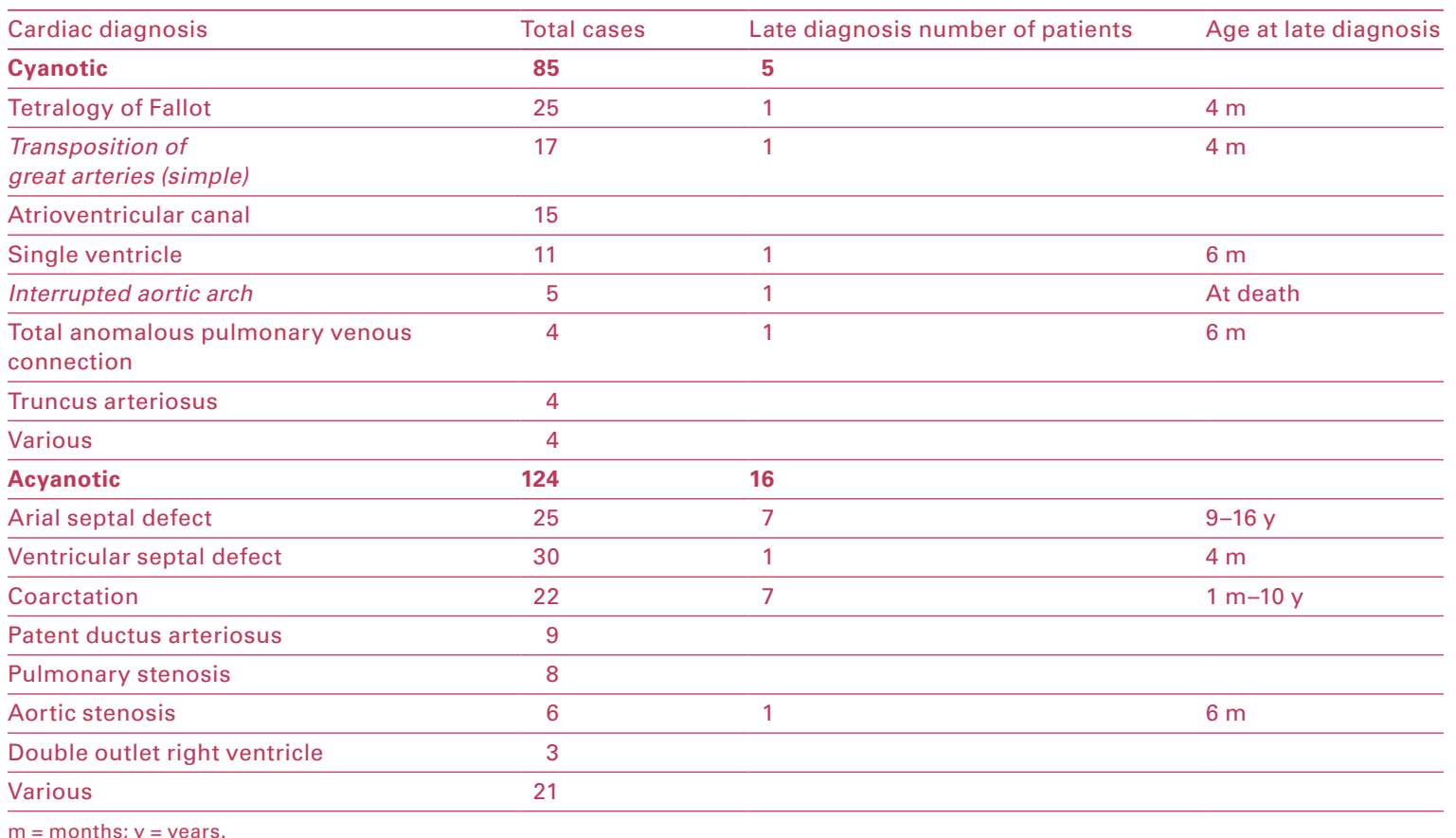


femoral pulses were the leading clinical sign in seven patients but were not detected until late diagnosis was made. Clearly present increased precordial pulsation on palpation of the thorax was noticed in four infants but was not reported before.

Only a few patients had clinical consequences as a result of late referral: there was one mortality due to late diagnosis of interrupted aortic arch with intractable cardiogenic shock at referral to the intensive care unit at 7 days of life. Two teenagers with an atrial septal defect (13 and 15 years old at referral and at device closure with an Amplatzer) did not normalise the size of their right heart structures during a 2-year follow-up.

\section{Discussion}

The main finding of this study was that in a contemporary cohort of children with newly diagnosed relevant CHD there was still a significant proportion of patients with late referral and late diagnosis of CHD. In comparison with an identical historical study published in 2001 [7], the overall rate of late diagnosis remained stable at $10 \%$ of patients with relevant CHD. The only difference was that there was a mild (although not significant) decline in late diagnosis of cyanotic CHD, whereas a slightly larger proportion of patients with acyanotic CHD were diagnosed late. The rise in the number of acyanotic patients diagnosed late was largely due to patients with coarctation and atrial septal defect. Late diagnosis of atrial septal defect even in the adult age is a constant finding in large populations undergoing echocardiographic studies [10] and thus not surprising. The mild decline in late referrals of cyanotic CHD patients is attributable to neonatal pulse oximetry screening (on the first day of life), although the remaining few patients not detected by screening make clear that in addition to pulse oximetry screening, a clinical examination still plays an important part as outlined in the recommendations accompanying the original screening guidelines [8].

The aim of a timely diagnosis of CHD is to minimise morbidity and mortality that may be associated with late referral $[3,5]$ and which may be relevant to the child's prognosis. Morbidity was low in the current study, but the case of a newborn who died because of a missed interruption of the aortic arch is alarming, this condition being amenable to surgical treatment with good long-term results.
Another asthonishing result of the current evaluation is the very low rate of antenatal echocardiographic diagnosis of relevant CHD, the detection rate of $19 \%$ being clearly below target standards in the current era [11], given the proven benefit of antenatal diagnosis in the management of critical CHD [12].

In summary, because during the past decade the rate of late detection of CHD did not show any decline, efforts to improve this performance have to continue on all levels, a higher detection rate of critical CHD already in utero as well as better recognition neonatally must be aimed for. Clinical recognition of atrial septal defects and coarctation must also be improved in order not to compromise the individual patient's prognosis.

\section{Disclosure statement}

No financial support and no other potential conflict of interest relevant to this article was reported.

\section{References}

1 Meberg A, Otterstad JE, Froland G, Hals J, Sörland SJ. Early clinical screening of neonates for congenital heart defects: the cases we miss. Cardiol Young. 1999;9:169-74.

2 Kuehl KS, Loffredo CA, Ferencz C. Failure to diagnose congenital heart disease in infancy. Pediatrics. 1999;103:743-7.

3 Brown KL, Ridout DA, Hoskote A, Verhulst L, Ricci M, Bull C. Delayed diagnosis of congenital heart disease worsens preoperative condition and outcome of surgery in neonates. Heart. 2006;92: 1298-302.

4 Massin MM, Dessy H. Delayed recognition of congenital heart disease. Postgrad Med J. 2006;82:468-70.

5 Chang RK, Gurvitz M, Rodriguez S. Missed diagnosis of critical con genital heart disease. Arch Pediatr Adolesc Med. 2008;162:969-74.

6 Koshnood B, Lelong N, Houyel L, Thieulin AC, Jouannic JM, Magnier S, et al. Prevalence, timing of diagnosis and mortality of newborns with congenital heart defects: a population - based study. Heart. 2012;98:1667-73.

7 Pfammatter JP, Stocker FP. Delayed recognition of hemodynamically relevant congenital heart disease. Eur J Pediatr. 2001;160: 213-4.

8 Arlettaz R. Recommendations for a neonatal pulsoxymetry screening for detection of congenital heart disease. Paediatrica. 2005;16:34-7.

9 Rao PS. Consensus on timing of intervention for common congenital heart diseases: part I - acyanotic defects. Indian J Pediatr. 2013; 80:663-74.

10 Eichhorn P, Sütsch G, Jenni R. Newly diagnosed congenital heart disease in adolescents and children. Swiss Med Wkly. 1990;120: 1697-700.

11 Van Velzen C, Clur S, Rijlaarsdam M, Bax C, Pajkrt E, Heymans M, et al. Prenatal detection of congenital heart disease - results of a national screening programme. BJOG. 2015; doi: 10.1111/14710528.13274 (Epub ahead of print).

12 Holland BJ, Myers JA, Woods CR. Prenatal diagnosis of critical congenital heart disease reduces risk of death from cardiovascular compromise prior to planned cardiac surgery: a meta analysis. Ultrasound Obstet Gynecol. 2015; 45(6):631-8. 